\title{
Alcaloide esteroidal, substância de Solanum paludosum, com atividade larvicida sobre Aedes aegypti
}

\author{
Steroidal alkaloid, Solanum paludosum substance, with larvicidal activity on Aedes aegypti
}

Igor Luiz Souza da Cruz ${ }^{* 1,2}$, Bárbara Mendes Pardal ${ }^{1}$, Simone Pereira Alves ${ }^{1}$, Gabriela Alves Melquíades de Medeiros $^{3}$, José Maria Barbosa-Filho ${ }^{3}$, Marise Maleck ${ }^{1,2,4,5}$

Como citar esse artigo. Cruz, I.L.S Pardal, B.M; Alves, S.P; de Medeiros, G.A.M; Barbosa-Filho, J.M; Maleck, M. Alcaloide esteroidal, substância de Solanum paludosum, com atividade larvicida sobre Aedes aegypti. Revista de Saúde. 2019 Jan./Jun.; 10 (1): 15-19.

\section{Resumo}

Aedes aegypti é reconhecido no Brasil como o vetor de importantes doenças de importância médica, como a dengue, zika, chikungunya e febre amarela urbana. Como o controle é a principal forma de se prevenir das afecções provocadas por este mosquito, os serviços de Saúde Pública fazem uso de inseticidas sintéticos químicos, que tendem a impactar o biossistema. Os produtos naturais obtidos de plantas se apresentam como uma alternativa menos tóxica e eficaz no controle de pragas e vetores. Neste estudo buscou-se avaliar a ação de Solasodina, isolada de Solanum paludosum, sobre as formas imaturas de A. aegypti. A substância foi aplicada no meio de criação das larvas (L3) nas concentrações de 10, 50,100 e $150 \mu \mathrm{g} / \mathrm{mL}$. O alcaloide interferiu no desenvolvimento do vetor, com um aumento considerável na duração do ciclo biológico. Solasodina apresentou toxidade considerável (70 \%) sobre as larvas do culicídeo na concentração de 150 $\mu \mathrm{g} / \mathrm{mL}$. Os dados mostraram atividade larvicida do alcaloide esteroidal solasodina e sua interferência no desenvolvimento de A. aegypti.

Palavras-chave: Culicidae, Produtos Naturais, Solasodina.

\begin{abstract}
Aedes aegypti is recognized in Brazil as the vector of important diseases of medical importance, such as dengue, zika, chikungunya and urban yellow fever. As control is the main way to prevent the diseases caused by this mosquito, the public health services make use of synthetic chemical insecticides, which tend to impact the bio system. The natural products obtained from plants are presented as a less toxic and effective alternative in pests and vector control. This study aimed to evaluate the action of solasodine, isolated from Solanum paludosum, on the immature forms of $A$. aegypti. The substance was applied to larvae (L3) at concentrations of 10, 50, 100 and $150 \mu \mathrm{g} / \mathrm{ml}$. The alkaloid interfered in the development of the vector, with a considerable increase in the development phase until the adult phase. Solasodine presented considerable larval toxicity $(70 \%)$ on culicide larvae at a concentration of $150 \mu \mathrm{g} / \mathrm{mL}$. The data showed larvicidal activity of the solasodine steroidal alkaloid and its interference in the development of $A$. aegypti.
\end{abstract}

Keywords: Culicidae, Natural products, Solasodine.

\section{Introdução}

A espécie Aedes (Stegomyia) aegypti (Linnaeus, 1762) é conhecida por ser o principal responsável pelo agravo da situação epidemiológica no Brasil. Este mosquito com hábitos oportunistas é vetor da dengue, presente em todo território nacional, dos vírus Chikungunya (CHIKV) e Zika (ZIKV), que foram introduzidos com eficiência no país, e capazes de transmitir o ciclo urbano da doença infecciosa aguda identificada como febre amarela (FA) ${ }^{1-4}$.

As principais estratégias utilizadas no controle do A. aegypti ainda são baseadas no controle a partir do uso de inseticidas químicos sintéticos, principalmente os organofosforados e o piretroides. Entretanto, a utilização inadvertida desses compostos representa um problema no aumento de populações resistentes na Ásia e na América Latina 5 .

Dentro desta proposta, os produtos naturais obtidos de plantas vêm se mostrando altamente eficazes, oferecendo uma nova perspectiva no combate a vetores. Estes compostos se apresentam como uma ótima alternativa de controle por serem menos agressivos ao meio ambiente ${ }^{6}$. Os mosquitos de importância médica, como o A. aegypti, são alvos de constantes pesquisas com derivados botânicos que apresentam ação inseticida e atuam no ciclo de desenvolvimento do culícideo ${ }^{7-8}$.

As plantas originam uma série de metabólitos especiais, como esteroides, terpenoides, alcaloides,

Afiliação dos autores: 1. Laboratório de Insetos Vetores, Universidade de Vassouras, Vassouras, Rio de Janeiro, Brasil.

2. Mestrado Profissional em Ciências Ambientais, Universidade de Vassouras, Vassouras, Rio de Janeiro, Brasil.

3. Instituto de Pesquisa em Fármacos e Medicamentos, Universidade Federal da Paraíba, João Pessoa, Paraíba, Brasil.

4. Pró-Reitoria de Ciências Médicas, Universidade de Vassouras, Vassouras, Rio de Janeiro, Brasil.

5. Laboratório de Entomologia Médica e Forense, IOC - FIOCRUZ, Rio de Janeiro, Rio de Janeiro, Brasil.

* Email de correpondência: igorlscruz@gmail.com

Recebido em: 07/05/19. Aceito em: 21/05/19. 
óleos essenciais, dentre outros, que apresentam diversas funções e aplicações, principalmente, na agricultura e na medicina ${ }^{9-10}$.

A espécie neotropical Solanum paludosum Moric, está amplamente distribuída pela América do Sul, e presente na Guiana, Guiana Francesa, Suriname, Venezuela e no Brasil ${ }^{11}$. Esta espécie faz parte da família Solanaceae, gênero Solanum, e é conhecida pela presença de alcaloides esteroidais. Conhecida popularmente como jurubeba-brava ou jurubebaroxa, os frutos desta planta são tóxicos e a infusão de suas raízes utilizadas na medicina popular para tratar distúrbios hepáticos ${ }^{1213}$. Dos frutos de $S$. paludosum foi isolado o alcaloide esteroidal solasodina ${ }^{14}$.

Este estudo avaliou o potencial larvicida da solasodina, isolado de $S$. paludosum, sobre formas imaturas de A. aegypti.

\section{Material e métodos}

\section{Isolamento da Solasodina}

O alcaloide esteroidal solasodina (Figura 1) foi isolado de $S$. paludosum, conforme metodologia de Barbosa-Filho et al. ${ }^{12}$ e Silva, Agra e Bhattacharyya ${ }^{15}$.

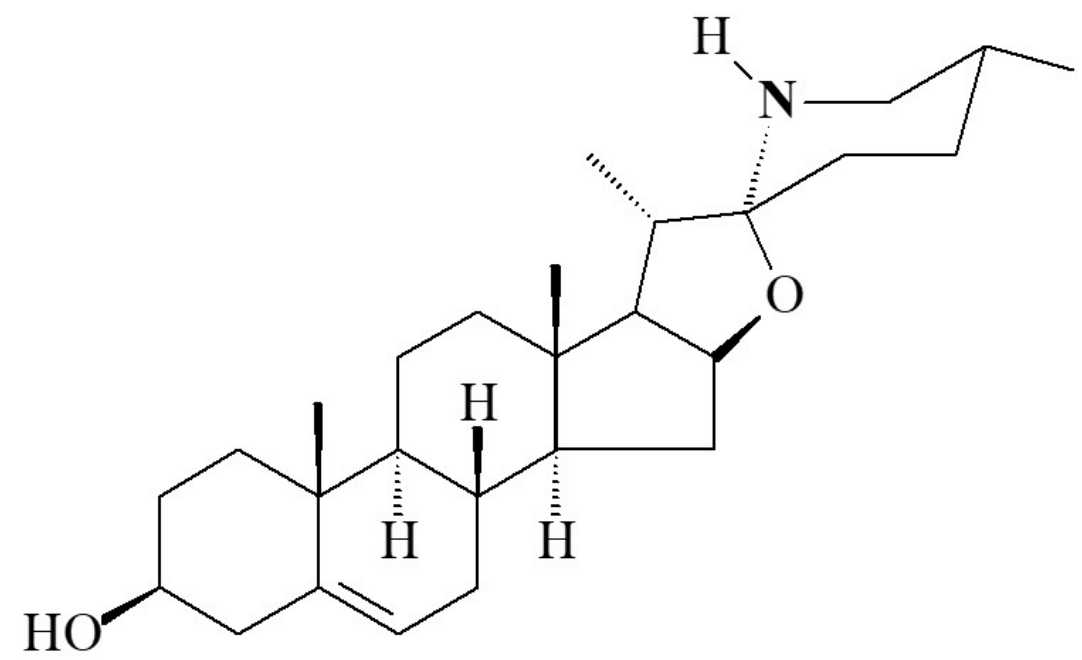

Figura 1. Solasodina, alcaloide esteroidal de Solanum paludosum Moric., Solanaceae. Adaptado de Barbosa-Filho et al. ${ }^{12}$

\section{Aedes aegypti}

Os ovos do mosquito A. aegypti L. 1762 foram obtidos a partir do Núcleo de Apoio às Pesquisas em Vetores (NAPVE) (parceria DIRAC-IOC-VPAAPS), Instituto Oswaldo Cruz, FIOCRUZ, Rio de Janeiro, Brasil. Para a realização dos bioensaios os ovos foram previamente analisados quanto a sua viabilidade e colocados para eclosão em recipientes contendo água mineral, aquecida antecipadamente, a $28^{\circ} \mathrm{C}$ nos quais foi adicionada a alimentação artificial de ração para peixe (Alcon Basic ${ }^{\circledR}$ ). Os recipientes (meio de criação) contendo os ovos foram mantidos em estufa incubadora do tipo BOD a $27 \pm 1{ }^{\circ} \mathrm{C}$ e $70 \pm 10 \%$ UR. Após a eclosão, as larvas de terceiro estádio (L3) foram separadas e selecionadas para os testes.

\section{Bioensaios}

Os bioensaios com solasodina foram realizados no Laboratório de Insetos Vetores, Universidade de Vassouras, Vassouras-RJ. A substância foi dissolvida em dimetilsulfóxido (DMSO) nas concentrações de $10,50,100,150 \mu \mathrm{g} / \mathrm{mL}$. Os testes foram realizados por aplicação $(\mu \mathrm{g} / \mathrm{mL})$ no meio de criação das larvas, em recipientes de vidro contendo água mineral $(20 \mathrm{~mL})$.

Foram dispostos seis grupos, cada um com 20 larvas de terceiro estádio (L3) de A. aegypti, de acordo com a World Health Organization ${ }^{16}$, sendo um grupo para controle (sem substância e sem DMSO), um grupo para controle testemunho (sem substância e com DMSO) e quatro grupos testes (com a substância e com DMSO). Os experimentos foram realizados em triplicatas (R1, 
R2 e R3), que totalizaram o uso de 60 larvas por grupo e foram feitas três repetições. Após a aplicação, as larvas foram mantidas em sua dieta normal, ração para peixe na proporção de $0,3 \mathrm{~g}$ para cada larva, em estufa incubadora (BOD a $27 \pm 1{ }^{\circ} \mathrm{C}$ e $70 \pm 10 \%$ UR) e foram avaliadas durante 35 dias tendo em consideração o seu desenvolvimento e sua mortalidade, de acordo com a metodologia de Maleck et al. ${ }^{17} \mathrm{e}$ de Serdeiro et al. ${ }^{18}$.

\section{Análise estatística}

Os resultados obtidos dos ensaios biológicos foram submetidos a análise de variância (ANOVA) e ao teste de Tukey, com nível de significância de 5\%, através do software MiniTab ${ }^{\circledR}$ 17.1.0. A análise estatística para o cálculo da dose letal $\left(\mathrm{DL}_{50}\right)$ foi realizada a partir do método Trimmed Spearman-Karbi ${ }^{19}$.

\section{Resultados}

O tratamento no meio de criação das larvas (L3) de $A$. aegypti com o alcaloide esteroidal solasodina, extraído de $S$. paludosum resultou em um aumento da permanência (em dias) do inseto nos períodos de desenvolvimento larval, pupal e de emergência. Na concentração de $10 \mu \mathrm{g} / \mathrm{mL}$, houve interferência na fase de pupa $(9,9 \pm 2,5$ dias; $\mathrm{p} \leq 0.01)$ e L3-Adulto $(12,5 \pm$ $2,4$ dias; $\mathrm{p} \leq 0.1)$ (Tabela 1A) e o mesmo ocorreu, de forma mais acentuada, com as concentrações de 100 $\mu \mathrm{g} / \mathrm{mL}$, fase de pupa $(11 \pm 4,3$ dias; $\mathrm{p} \leq 0.001)$ e L3Adulto $(15,9$ dias $\pm 3,8 \mathrm{p} \leq 0.001)$, e $150 \mu \mathrm{g} / \mathrm{mL}$, fase de pupa $(12,4 \pm 4,2$ dias; $p \leq 0.001)$ e L3-Adulto $(15,6 \pm$ $4,5$ dias; $p \leq 0.001)$. Apenas a concentração de $50 \mu \mathrm{g} / \mathrm{mL}$ demonstrou alteração significativa para todas as fases de desenvolvimento.

Tabela 1. Duração do desenvolvimento (A) e mortalidade (B) de Aedes aegypti submetidos ao tratamento com o alcaloide Solasodina, extraído de Solanum paludosum.

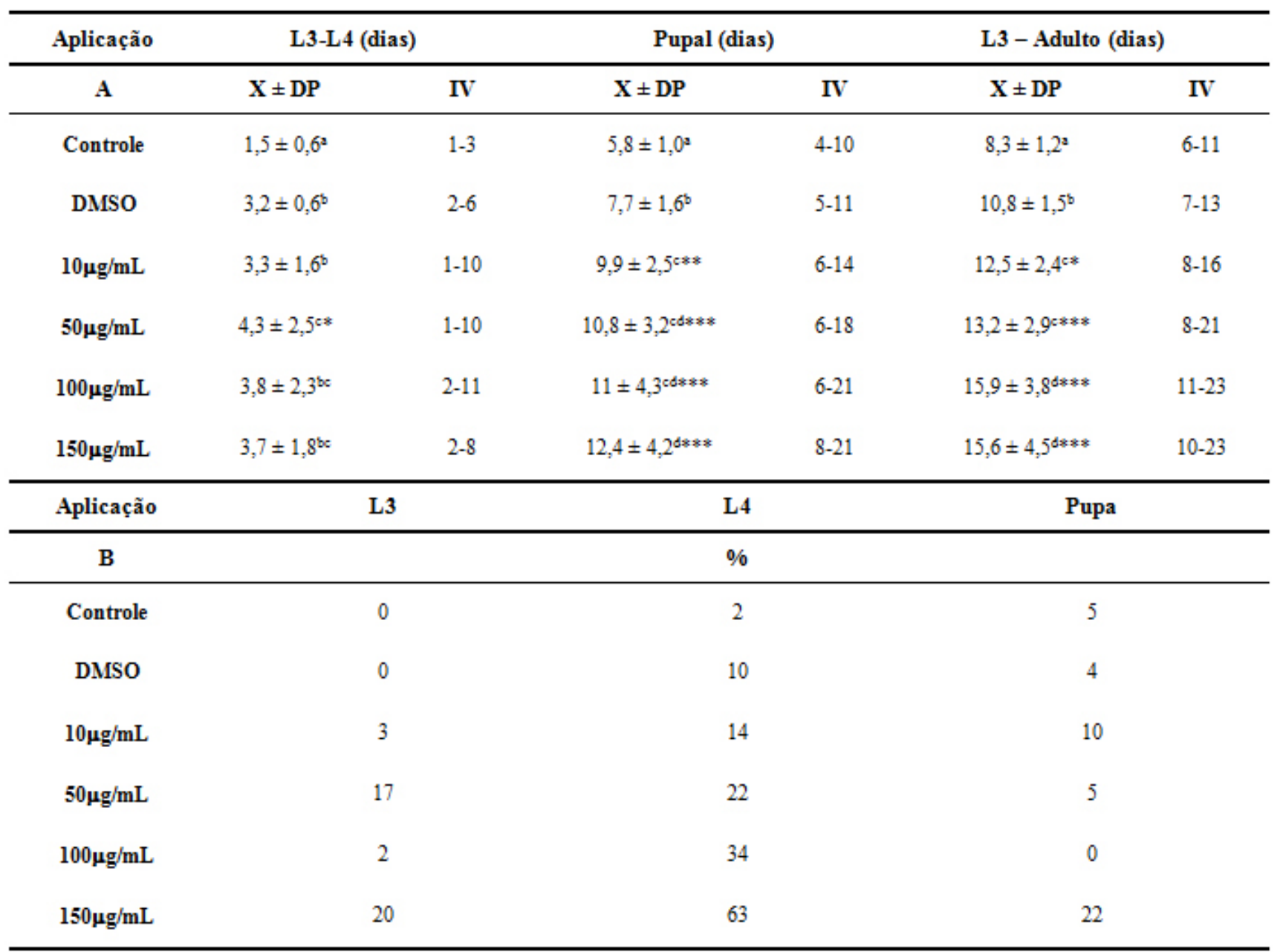

- Experimentos com 20 larvas (L3) de A. aegypti, para cada grupo teste e controle, em triplicatas e com 3 repetições. Média e desvio padrão (X \pm DP). Intervalo de Variação (IV). Valores seguidos da mesma letra não possuem diferenças significativas. Níveis de significância por teste de Tukey, representados como ${ }^{* * *} \mathrm{p} \leq 0.001 ;{ }^{*} \mathrm{p} \leq 0.01 ;{ }^{*} \mathrm{p} \leq 0.1$ vs controle DMSO (testemunho). 
$\mathrm{O}$ alcaloide evidenciou uma mortalidade de L3, na concentração de $150 \mu \mathrm{g} / \mathrm{mL}$, de $20 \%$. Nas larvas de quarto estádio, a solasodina apresentou mortalidade significativa em $50 \mu \mathrm{g} / \mathrm{mL}(22 \%), 100 \mu \mathrm{g} / \mathrm{mL}(34 \%)$ e $150 \mu \mathrm{g} / \mathrm{mL}(63 \%)$, e uma dose letal $\left(\mathrm{DL}_{50}\right)$ de $109 \mu \mathrm{g} /$ $\mathrm{mL}$.

\section{Discussão}

Diferentes compostos e substâncias da família Solanaceae vêm sendo isolados e investigados quanto à suabioatividade eação farmacológica. Estudos anteriores já demonstraram a utilização de extratos obtidos da espécie S. paludosum no tratamento de células tumorais e na modulação da resposta de resistência bacteriana a medicamentos ${ }^{20}$. Em outro momento, testes com plantas do gênero Solanum evidenciaram a resposta moluscicida das folhas e frutos (de diferentes espécies, incluindo $S$. paludosum) sobre Biomphalaria glabrata, hospedeiro intermediário do Schistosoma mansoni e causador da doença esquistossomose ${ }^{21}$.

Estudos que demonstrem a utilização de solasodina em insetos são incomuns, principalmente em dípteros. Entretanto, testes com larvas de besouroda-farinha (Tribolium confusum), considerado uma praga agrícola, já foram evidenciados e comprovaram a eficácia deste composto no desenvolvimento do inseto ${ }^{22}$. Essa interferência foi presenciada também neste estudo, em que o tempo (em dias) de evolução foi retardado, do estádio de L3 para a fase adulta nos grupos tratados com $\mathrm{o}$ alcaloide esteroidal.

$O$ potencial larvicida de plantas do gênero Solanum sobre A. aegypti já foi constatado. Larvas do mosquito foram expostas incessantemente por $72 \mathrm{~h}$ à extratos aquosos de Solanum villosum, na concentração de $0,5 \%$, o que revelou uma correlação entre o nível de mortalidade e o período de exposição ${ }^{23}$. Outros testes com A. aegypti utilizando extratos da espécie Solanum trilobatum demonstraram alteração na viabilidade de adultos, na concentração de $250 \mu \mathrm{g} / \mathrm{mL}$, com um índice de emergência de adultos de $42 \%$ (extrato acetônico), $28 \%$ (extrato clorofórmico) e $9 \%$ (extrato metanólico) ${ }^{24}$. Estes dados se alinham aos do presente estudo que demonstrou um reduzido número de emergência, após o tratamento com a substância na concentração de 150 $\mu \mathrm{g} / \mathrm{mL}$.

\section{Conclusão}

O alcaloide solasodina obtido de $S$. paludosum provocou alterações sobre o desenvolvimento de $A$. aegypti. A atividade larvicida foi expressivamente demonstrada com o resultado de $63 \%$ de mortalidade larval (L4) de A. aegypti. Este estudo mostrou através do alcaloide esteroidal o possível potencial de $S$. paludosum quanto a sua utilização no controle alternativo do $A$. aegypti.

\section{Agradecimentos}

Os autores agradecem o suporte financeiro do Conselho Nacional de Desenvolvimento Científico e Tecnológico (CNPq); da Fundação de Amparo à Pesquisa do Estado do Rio de Janeiro (FAPERJ) no projeto "Novas estratégias para o controle do mosquito Aedes aegypti, vetor da Dengue, Chikungunya e do virus Zika: uma abordagem integrada/Rede ZIKA\#I"; e à Fundação Educacional Severino Sombra (FUSVE).

\section{Referências}

1. Souza KR, Santos, MLR, Guimarães ICS, Ribeiro GS, Silva LK. Saberes e práticas sobre controle do Aedes aegypti por diferentes sujeitos sociais na cidade de Salvador, Bahia, Brasil. Cad Saúde Pública 2018;34(5); Article ID e00078017.

2. Lourenço AF, Rodrigues FM. Doenças transmitidas pelo Aedes aegypti (Linnaeus, 1762) no Brasil nos últimos dez anos. Estudos (Goiânia) 2017;44:72-77.

3. Luz KG, Santos GIV, Vieira RM. Febre pelo vírus Zika. Epidemiol Sery Saúde 2015;24(4):785-788.

4. Cavalcante KRLJ, Tauil PL. Risco de reintrodução da febre amarela urbana no Brasil. Epidemiol Serv Saude 2017;26(3):617-620.

5. Tomé HVV, Pascini TV, Dângelo RAC, Guedes RNC, Martins GF. Survival and swimming behavior of insecticide-exposed larvae and pupae of the yellow fever mosquito Aedes aegypti. Parasite Vector 2014;7:195.

6. Dietrich F, Strohschoen AAG, Schultz G, Sebben AD, Claudete Rempel C. Utilização de inseticidas botânicos na agricultura orgânica de Arroio do Meio/RS. R Bras Agrociência 2011; 17(2-4):p.251-255.

7. Viana GA, Sampaio CG, Pessoa VE, Martins VEP. Produtos naturais de origem vegetal como ferramentas alternativas para o controle larvário de Aedes aegypti e Aedes albopictus. J Health Biol Sci 2018;6(4):449-462.

8. Shaalan EA, Canyon D, Younes MW, Abdel-Wahab H, Mansour AH. A review of botanical phytochemicals with mosquitocidal potential. Environ Int 2005;31(8):1149-1166.

9. Chellappandian M, Vasantha-Srinivasan P, Senthil-Nathan S, Karthi S, Thanigaivel A, Ponsankar A, Kalaivani K, Hunter WB. Botanical essential oils and uses as mosquitocides and repellents against dengue. Environ Int 2018;113:214-230.

10. Pinto AC, Silva DHS, Bolzani VS, Lopes NP, Epifanio RA. Produtos naturais: atualidade, desafios e perspectivas. Quim Nova 2002;25:45-61.

11. Basílio IJLD, Agra MF, BhattacharyyaJ. Estudo farmacobotânico de folhas de

Solanum paludosum Moric. (Solanaceae). Rev Bras Biociênc 2007;5:651653.

12. Barbosa-Filho JM, Agra MF, Oliveira RA, Paulo MQ, Trolin G, Cunha EV, Ataide JR, Bhattacharyya J. Chemical and pharmacological investigation of Solanum species of Brazil--a search for Solasodine and other potentially useful therapeutic agents. Mem Inst Oswaldo Cruz 1991;86:189-191.

13. Agra MF, Freitas PF, Barbosa-Filho JM. Synopsis of the plants known as medicinal and poisonous in Northeast of Brazil. Rev Bras Farmacogn 2007;17(1):114-140.

14. Bhattacharyya J. Isolation of Solasodine from the fruits of Solanum asperum and Solanum paludosum. J Nat Prod 1984;47(6):10591060. 
15. Silva TMS, Agra MF, Bhattacharyya, J. Studies on the alkaloids of Solanum of northeastern Brazil. Rev Bras Farmacogn 2005;15(4):292-293.

16. World Health Organization. 2005. Guidelines for laboratory and field testing of mosquito larvicides. Disponível em: http://apps.who.int/iris/ bitstream/10665/69101/1/

17. Maleck M, Hollanda PO, Serdeiro MT, Soares RO, Honório NA, Silva CG. Toxicity and larvicidal activity of Podophyllum-based lignans against Aedes aegypti (Diptera: Culicidae). J Med Entomol 2017;54(1):159-66.

18. Serdeiro MT, Mallet JRS, Honório NA, Maleck M. Aedes aegypti: modelo experimental de atividade biológica de fitoprodutos. R Saúde 2017;8(1):2832 .

19. Hamilton MA, Russo RC, Thurson RV. Trimmed Spearman-Karber Method for Estimating Median Lethal Concentrations in Toxicity Bioassays. Environ Sci Technol 1977;11(7):714-719.

20. Siqueira S, Falcão-Silva VS, Agra MF, Dariva C, Siqueira-Júnior JP, Fonseca MJV. Biological activities of Solanum paludosum Moric. extracts obtained by maceration and supercritical fluid extraction. J Supercrit Fluid 2011;58:391-397.

21. Silva TMS, Batista MM, Camara CA, Agra MF. Molluscicidal activity of some Brazilian Solanum spp. (Solanaceae) against Biomphalaria glabrata. Ann Trop Med Parasit 2005;99(4):419-425.

22. Lingampally V, Solanki VR, Anuradha DL, Raja SS. Effect of Solasodine against Last Instar Larvae of Tribolium confusum. J Entomol Zool Stud 2014;2(3):118-120.

23. Chowdhury N, Ghosh A, Chandra G. Mosquito larvicidal activities of Solanum villosum berry extract against the dengue vector Stegomyia aegypti. BMC Complement Altern Med 2008;8(1):10.

24. Premalatha S, Elumalai K, Jeyasankar A. Mosquitocidal properties of Solanum trilobatum L. (Solanaceae) leaf extracts against three important human vector mosquitoes (Diptera: Culicidae). Asian Pac J Trop Med 2013;6(11):854-858. 